\title{
Características do acesso de crianças com microcefalia aos serviços de fisioterapia
}

\author{
Characteristics of access of children with \\ microcephaly to physical therapy services
}

\author{
Danyelle Nóbrega de Farias ${ }^{1}$ (1) \\ Robson da Fonseca Neves ${ }^{2}$ (1) \\ Geraldo Eduardo Guedes de Brito 3 (1) \\ Emanuelle Silva de Mélo ${ }^{4}$ (i)
}

\section{Larissa Duarte de Britto Lira 5 (1) Letícia Mylena Guedes Souza ${ }^{6}$ (i) João Agnaldo do Nascimento7 (1) Kátia Suely Queiroz Silva Ribeiro 8 (1)}

\footnotetext{
${ }^{1}$ Autora para correspondência. Universidade Federal da Paraíba (Paraíba). Paraíba, Brasil. danynobregadefarias@hotmail.com ${ }^{2-8}$ Universidade Federal da Paraíba (Paraíba).Paraíba, Brasil. robsonfisioba@gmail.com, dudugjf@yahoo.com.br, eman_melo27@hotmail.com, larissadblira@hotmail.com, leeticiamylena@gmail.com, joaoagh@gmail.com, katiaqsribeiro@yahoo.com.br
}

RESUMO | INTRODUÇÃO: A "síndrome congênita do Zika" compreende um conjunto de sinais e sintomas apresentados por crianças nascidas de mães infectadas durante a gestação. Os RN acometidos por microcefalia apresentam alterações no desenvolvimento neuropsicomotor, assim, o processo de reabilitação torna-se fundamental para o desenvolvimento das crianças. OBJETIVO: Nessa perspectiva, faz-se necessário considerar o acesso em seu domínio amplo, e compreender as características do acesso de crianças com microcefalia nascidas nos anos de 2015 e 2016 aos serviços de fisioterapia. MÉTODOS: Trata-se de um estudo transversal, de abordagem quantitativa, com caráter descritivo. Utilizou-se um questionário validado por quatro especialistas, coletando informações acerca da caracterização socioeconômica e clínica, identificação do tempo entre o encaminhamento das crianças e sobre as características do acesso à reabilitação. RESULTADOS: A amostra deste estudo foi composta por 103 cuidadores de crianças com microcefalia. Observou-se que $93,2 \%$ das crianças tiveram encaminhamento para procurar o serviço de fisioterapia e buscaram atendimento, entretanto, nem todas as crianças mantiveram-se em reabilitação. As crianças que tiveram acesso continuado e as que descontinuaram o cuidado em algum momento apresentavam-se de forma semeIhante no que diz respeito à frequência de tratamento, realização da fisioterapia em mais de um serviço, tempo de duração da sessão, despendimento de pagamento para exames e interferência na atividade profissional com o tratamento da criança. CONCLUSÃo: As que se mantiveram em acesso sem interrupções, possuíam um auxílio maior do município para deslocamento, além da oportunidade de assistência da rede de apoio ao cuidador, potencializando a manutenção do cuidado.

PALAVRAS-CHAVE: Acesso aos serviços de saúde. Reabilitação. Microcefalia. Fisioterapia.
ABSTRACT | INTRODUCTION: The "congenital Zika syndrome" comprises a set of signs and symptoms presented by children born to infected mothers during pregnancy. Newborns affected by microcephaly present alterations in their neuropsychomotor development. Therefore, the rehabilitation process becomes essential for the development of children. OBJECTIVE: From this perspective, it is necessary to consider access in its broad domain and understand the characteristics of children with microcephaly (born in 2015 and 2016) access to physical therapy services. METHODS: This is a cross-sectional study, with a quantitative approach, with a descriptive character. A questionnaire validated by four experts was used, collecting information about the socioeconomic and clinical characteristics, identification of the time between the referral of children, and the characteristics of access to rehabilitation. RESULTS: The sample of this study consisted of 103 caregivers of children with microcephaly. It was observed that $93.2 \%$ of the children were referred to seek the physiotherapy service and searched for it. However, not all children remained in rehabilitation. Children who had continuous access and those who discontinued care at some point similarly presented themselves concerning the frequency of treatment, physical therapy in more than one service, duration of the session, expenditure of payment for exams, and interference in the professional activity with the child's treatment. CONCLUSION: Those who remained in uninterrupted access had better assistance from the municipality for displacement, in addition to the opportunity for help from the caregiver support network, enhancing the maintenance of care.

KEYWORDS: Access to health services. Rehabilitation. Microcephaly. Physiotherapy. 


\section{Introdução}

A partir da epidemia do vírus Zika no Brasil e do crescimento de casos de crianças com microcefalia, no ano de 2015, estudos foram realizados para investigar a associação desta infecção com malformações em recém-nascidos (RN), sobretudo da microcefalia. ${ }^{1}$

A microcefalia pode ser diagnosticada ainda durante a gestação, no acompanhamento por meio de exames pré-natais, ou pela avaliação clínica de rotina a que são submetidos os recém-nascidos nas primeiras 24 horas de vida. Neste caso, realiza-se a aferição do Perímetro Cefálico (PC), com a medição da Circunferência Occipital-Frontal (COF), chegando ao diagnóstico da microcefalia quando o valor for menor do que o esperado para a idade gestacional e sexo. $O$ fato de se observar um crescimento inadequado do crânio leva a conclusão de que o cérebro se encontra subdimensionado, logo, apresentando aspectos anatomofisiológicos que fogem da normalidade. 1

A epidemia da "síndrome congênita do Zika" compreende um conjunto de sinais e sintomas apresentados por crianças nascidas de mães infectadas por esse vírus durante a gestação, sendo a microcefalia a alteração mais marcante do Sistema Nervoso Central associada à infecção pelo vírus. Os RN acometidos por microcefalia têm alterações no desenvolvimento neuropsicomotor, podendo apresentar, também, deficiência intelectual, epilepsia, paralisia cerebral, déficit cognitivo, comprometimentos motores e na fala. Também podem estar presentes problemas de visão e de audição.?

Desde a publicação do Decreto 7.692 de 17/11/2011 e da Portaria 793/2012 que instituem o Plano Nacional da Pessoa com Deficiência - Plano Viver sem Limites e a Rede de Cuidados à Pessoa com Deficiência no SUS (RCPCD), respectivamente, teve início um movimento de expansão dos serviços de reabilitação no país. As novas demandas decorrentes do aumento súbito no número de crianças com microcefalia, no período supracitado, demandaram uma reorganização da rede de serviços, no sentido de atender às necessidades de cuidado impostas por esse agravo e o fato de que a RCPCD estivesse em expansão foi um elemento favorável, no entanto, a RCPCD, naquele momento, ainda estava em fase inicial de implantação. Estas demandas incluem ações diagnósticas e terapêuticas, com ênfase para o acompanhamento especializado ao desenvolvimento destas crianças, visando prevenir e/ou minimizar sequelas decorrentes desta condição.

Nesse contexto, o processo de reabilitação torna-se fundamental para o desenvolvimento dos bebês e deve ser iniciado o mais breve possível, levando em consideração as inúmeras sequelas apresentadas em decorrência da má formação cerebral. 3

O acesso é definido aqui como a utilização de um serviço a tempo de se obter uma reabilitação satisfatória e continuidade no processo de tratamento. ${ }^{4}$ Mais que isso, conforme assinalam Travassos e Viacava ${ }^{5}$, o acesso reflete as características do sistema de saúde, que atuam aumentando ou diminuindo barreiras à obtenção de serviços pela população. Esses autores advogam ainda, que, a utilização de serviços de saúde pode ser entendida como a entrada nos mesmos, sendo uma expressão positiva do acesso.

Com base na definição do fluxo de eventos entre necessidades de saúde e a obtenção de cuidados, três domínios são propostos para o acesso ${ }^{6}$, conforme diagrama abaixo: 
Figura 1. Fluxo de eventos entre necessidades de saúde e a obtenção de cuidados, adaptado de Frenk. 으 $^{-}$

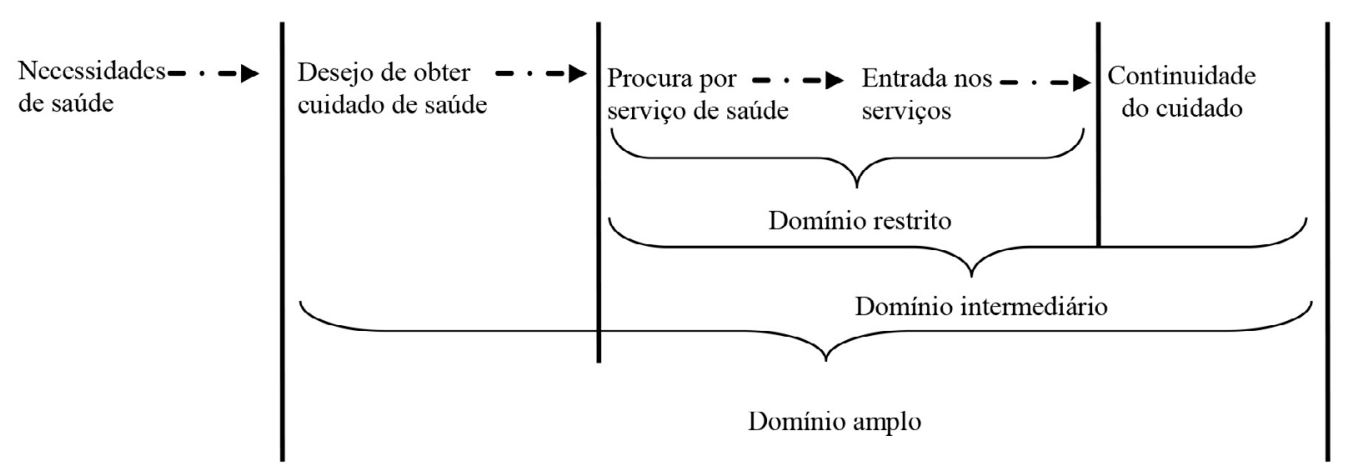

Ressalta-se, assim que, considerar o acesso apenas pela entrada nos serviços corresponde à dimensão restrita do acesso. No presente estudo, adotar-se-á como referência a perspectiva ampliada do acesso, a qual inclui não apenas a entrada no serviço, mas a continuidade do cuidado. A escolha por esta referência se justifica pela especificidade do processo de reabilitação, que requer uma permanência da pessoa em tratamento por períodos longos, sobretudo quando voltada aos comprometimentos neurológicos. Assim, o objetivo deste estudo é considerar o acesso em seu domínio amplo, e compreender as características do acesso de crianças com microcefalia nascidas nos anos de 2015 e 2016 aos serviços de fisioterapia em uma capital do Nordeste. Espera-se, a partir daí, contribuir para aprimorar o processo de organização da Rede.

\section{Método}

Trata-se de um estudo transversal, de abordagem quantitativa, com caráter descritivo. Foram incluídos todos os dados de acesso das crianças diagnosticadas com microcefalia, nascidas entre os anos de 2015 e 2016, com possível associação ao Zika Vírus no estado da Paraíba, constantes na lista disponibilizada pela Secretaria Estadual de Saúde (SES), a partir de notificações registradas no Formulário de Registro de Eventos de Saúde Pública (RESP) - Microcefalia, totalizando uma população de 191 crianças.

Utilizou-se um questionário, elaborado a partir de criteriosa revisão da literatura e validado por quatro especialistas, coletando informações acerca da caracterização socioeconômica e clínica, identificação do tempo entre o encaminhamento das crianças e o acesso à reabilitação. Este instrumento foi aplicado aos cuidadores das crianças com microcefalia.

A coleta de dados se iniciou por meio da participação dos pesquisadores na Caravana do Coração, um projeto que foi fruto do convênio entre o Círculo do Coração de Pernambuco e o Governo do Estado da Paraíba, com o objetivo de realizar triagens, exames e consultas de acompanhamento de crianças em 13 municípios do Estado da Paraíba. A parceria com esse projeto do governo estadual possibilitou a realização da coleta em todas as regiões de saúde do Estado da Paraíba, a mesma ocorreu nos anos de 2017 e 2018.

Assim, o rastreio e a coleta das informações das crianças foram realizados a partir dos serviços de referência para reabilitação e participaram do estudo todas as crianças com diagnóstico de microcefalia, nascidas entre os anos de 2015 e 2016, cujas mães tinham histórico de infecção pelo Vírus Zika. A amostra final desse estudo ficou em 103 crianças com microcefalia associada ao vírus Zika, cujos pais ou responsáveis foram entrevistados. 


\section{Resultados}

Para a apresentação dos resultados referentes à caracterização do acesso, foi utilizada a estatística descritiva. O estudo foi submetido à avaliação pelo Comitê de Ética em Pesquisa, do Centro de Ciências da Saúde, da Universidade Federal da Paraíba (CAAE: 64800416.9.1001.5188). Todos os participantes assinaram o Termo de Consentimento Livre e Esclarecido como rege a Resolução 466/12 do Conselho Nacional de Saúde.

A amostra deste estudo foi composta por 103 cuidadores de crianças com microcefalia. A maioria das mães das crianças deste estudo tinha mais de 21 anos (82,6\%), já estavam na terceira gestação ou mais $(35,9 \%)$ e referiram não possuir Ensino Médio (96,2\%). Uma extensa parcela dos entrevistados relatou estar inativa $(64,1 \%)$ e maioria declarou estar casada ou em união estável $(69,9 \%)$, como se pode observar na tabela 1.

A maioria das crianças nasceu com perímetro cefálico abaixo de 31 centímetros $(83,4 \%)$, o perímetro cefálico da criança ao nascer foi uma variável importante a fim de constatar o diagnóstico tardio. Aproximadamente metade das entrevistadas $(48,5 \%)$ havia descoberto o diagnóstico da criança antes do parto; mais da metade $(51,5 \%)$ descobriu no parto ou pós-parto e não apresentou quaisquer complicações durante a gravidez (73,8\%). A maioria relatou fazer uso do Benefício da Prestação Continuada (BPC) $(85,4 \%)$, e ter renda domiciliar entre um e dois salários-mínimos (99\%). Com relação à necessidade de encaminhamento à UTI logo ao nascer, 20,4\% das crianças foram internadas e possuem como cuidadora principal a mãe, 91,3\%, descritos na tabela 2 .

Quase a totalidade das crianças, 93,2\%, teve algum encaminhamento para procurar o serviço de fisioterapia. A maioria dos cuidadores, $88,3 \%$, declarou ter iniciado o processo de reabilitação com até oito semanas após o encaminhamento, $71,9 \%$.
Destes, $74,7 \%$ fazem o tratamento fisioterapêutico no centro de reabilitação, e sem custos pessoais, 96,1\%, apontados na tabela 3.

Em síntese, as crianças eram atendidas, em sua maioria, nos Centros Especializados de Reabilitação, sem custos, entre 2 a 3 vezes na semana, com uma duração de 40 a 60 minutos, e grande parte referiu que o processo de tratamento interferiu em sua ocupação profissional anterior.

Salienta-se, contudo, que nem todos tiveram um processo de reabilitação continuado $(11,7 \%)$, o que pode ser evidenciado na tabela 4.

No que diz respeito às diferenças entre as crianças que tiveram seu tratamento interrompido em relação às que não tiveram interrupção, observa-se que ambos os grupos de crianças, apresentavam-se de forma semelhante no que diz respeito à frequência de tratamento, realização da fisioterapia em mais de um serviço, tempo de duração da sessão, despesas com exames. Em se tratando do deslocamento para realização do tratamento em outro município também não houve diferença entre as crianças que tiveram seu tratamento interrompido e as que se mantiveram em tratamento sem interrupção.

Todavia, verificou-se que as crianças que se mantiveram em acesso sem interrupções, possuíam um auxílio maior do município para deslocamento, ao contrário do grupo que em algum momento interrompeu a fisioterapia. A diferença também acontece no que concerne ao apoio/assistência ao cuidador, uma vez que mais da metade das mães que permaneceram com seus filhos em atendimento tiveram algum tipo de assistência, e das mães que descontinuaram o processo de reabilitação não obtiveram quaisquer oportunidades de assistência. 
Tabela 1. Caracterização sociodemográfica dos sujeitos do estudo

\begin{tabular}{|c|c|c|}
\hline \multirow[b]{2}{*}{ Variável } & \multicolumn{2}{|c|}{ Caracterização das mães das crianças com Microcefalia } \\
\hline & $(\mathrm{N})$ & $\%$ \\
\hline \multicolumn{3}{|l|}{ Faixa Etária } \\
\hline Até 20 anos & 18 & 17,5 \\
\hline Entre 21 e 30 Anos & 46 & 44,7 \\
\hline 30 anos ou mais & 39 & 37,9 \\
\hline \multicolumn{3}{|l|}{ Número de gestações } \\
\hline$\overline{1}$ & 30 & 29,1 \\
\hline 2 & 34 & 35,0 \\
\hline 3 ou mais & 39 & 35,9 \\
\hline \multicolumn{3}{|l|}{ Escolaridade } \\
\hline Ensin Fund. Incompleto & 34 & 35,0 \\
\hline Ensino Fund. Completo & 63 & 61,2 \\
\hline Ensino Médio & 3 & 1,92 \\
\hline Ensino Superior & 3 & 1,92 \\
\hline \multicolumn{3}{|l|}{ Ocupação } \\
\hline Ativo & 39 & 35,9 \\
\hline Inativo & 64 & 64,1 \\
\hline \multicolumn{3}{|l|}{ União Estável } \\
\hline Sim & 72 & 69,9 \\
\hline Não & 31 & 30,1 \\
\hline
\end{tabular}

Fonte: Dados da pesquisa, 2019

Tabela 2. Necessidades de saúde, clínica e social das crianças do estudo.

\begin{tabular}{|c|c|c|}
\hline Variável & $(\mathrm{N})$ & $\%$ \\
\hline \multicolumn{3}{|l|}{ Faixa Etária } \\
\hline Até 24 meses & 53 & 51,5 \\
\hline Mais de 24 meses & 50 & 48,5 \\
\hline \multicolumn{3}{|c|}{ Perímetro cefálico ao nascer $(\mathrm{cm})$} \\
\hline Entre 20 e 28 & 30 & 29,1 \\
\hline Entre 28 e 31 & 56 & 54,3 \\
\hline Acima de 31 & 4 & 3,9 \\
\hline Não sabe & 13 & 12,7 \\
\hline \multicolumn{3}{|l|}{ Diagnóstico } \\
\hline Antes do parto & 43 & 41,7 \\
\hline No parto & 10 & 9,7 \\
\hline Pós parto & 50 & 48,5 \\
\hline \multicolumn{3}{|c|}{ Complicações durante a gravidez } \\
\hline Sim & 27 & 26,2 \\
\hline Não & 76 & 73,8 \\
\hline \multicolumn{3}{|c|}{ Auxílio destinado a condição } \\
\hline Sim & 88 & 85,4 \\
\hline Não & 15 & 14,6 \\
\hline \multicolumn{3}{|c|}{ Renda da família que cuida da criança (salários mínimos/mês) } \\
\hline Até um & 1 & 1,0 \\
\hline Entre um e dois & 101 & 98,0 \\
\hline Mais de dois & 1 & 1,0 \\
\hline \multicolumn{3}{|l|}{ Realizou pré natal } \\
\hline Sim & 100 & 98,0 \\
\hline Não & 3 & 1,92 \\
\hline \multicolumn{3}{|c|}{ Encaminhamento para UTI logo ao nascer } \\
\hline Sim & 21 & 20,4 \\
\hline Não & 82 & 79,6 \\
\hline
\end{tabular}

Fonte: Dados da pesquisa, 2019. 
Tabela 3. Condições de acesso à Fisioterapia: procura e entrada nos serviços de reabilitação

\begin{tabular}{|c|c|c|}
\hline \multirow[b]{2}{*}{ Variável } & \multicolumn{2}{|c|}{ Condições de saúde da criança e de acesso à Fisioterapia } \\
\hline & (N) & $\%$ \\
\hline \multicolumn{3}{|c|}{ Encaminhamento ao Serviço de Fisioterapia } \\
\hline Sim & 96 & 93,2 \\
\hline Não & 7 & 6,8 \\
\hline \multicolumn{3}{|c|}{ Acesso ao serviço de Reabilitação } \\
\hline $\operatorname{Sim}$ & 92 & 88,3 \\
\hline Sim, mas interrompeu & 11 & 11,7 \\
\hline \multicolumn{3}{|c|}{ Tempo que a criança levou para ter acesso ao serviço de Fisioterapia } \\
\hline Até 2 semanas & 39 & 37,9 \\
\hline Entre 3 e 8 semanas & 35 & 34,0 \\
\hline Mais de 8 semanas & 29 & 28,2 \\
\hline \multicolumn{3}{|c|}{ Local que realiza/realizou o tratamento } \\
\hline Domicílio & 2 & 2,0 \\
\hline Centro de Reabilitação & 77 & 74,7 \\
\hline Hospital & 5 & 4,9 \\
\hline Clínica Escola & 19 & 18,4 \\
\hline \multicolumn{3}{|l|}{ O tratamento é pago } \\
\hline Sim & 4 & 3,9 \\
\hline Não & 99 & 95,9 \\
\hline
\end{tabular}

Fonte: Dados da pesquisa, 2019.

Tabela 4. Dados de continuidade do cuidado nos serviços de Fisioterapia

\begin{tabular}{|c|c|c|c|c|}
\hline \multirow[t]{2}{*}{ Variável } & \multicolumn{2}{|c|}{ Acesso Continuado } & \multicolumn{2}{|c|}{ Acesso Descontinuado } \\
\hline & $\mathbf{N}$ & $\%$ & $\mathbf{N}$ & $\%$ \\
\hline \multicolumn{5}{|c|}{ Realização do tratamento no mesmo município de residência da família } \\
\hline Sim & 40 & 44,0 & 6 & 54,6 \\
\hline Não & 42 & 46,1 & 3 & 27,3 \\
\hline Em mais de um município & 9 & 9,9 & 2 & 18,1 \\
\hline \multicolumn{5}{|c|}{ Auxilio do município em que reside para se deslocar } \\
\hline Sim & 52 & 57,1 & 4 & 36,4 \\
\hline Não & 7 & 7,7 & 2 & 18,2 \\
\hline Não se aplica & 32 & 35,2 & 5 & 45,4 \\
\hline \multicolumn{5}{|l|}{ Tempo de tratamento (anos) } \\
\hline Menos de 1 ano & 18 & 17,5 & - & - \\
\hline Mais de 2 & 85 & 82,5 & - & - \\
\hline \multicolumn{5}{|c|}{ Frequência do tratamento (dias por semana) } \\
\hline 1 & 17 & 18,7 & 3 & 27,3 \\
\hline 2 & 38 & 41,7 & 5 & 45,4 \\
\hline 3 & 20 & 22,0 & 2 & 18,2 \\
\hline Mais de 3 & 16 & 17,6 & 1 & 9,1 \\
\hline \multicolumn{5}{|c|}{ Se a fisioterapia é realizada em mais de um serviço } \\
\hline Sim & 54 & 59,3 & 1 & 9,1 \\
\hline Não & 36 & 39,6 & 10 & 90,9 \\
\hline Não sabe & 1 & 1,1 & - & - \\
\hline \multicolumn{5}{|c|}{ Tempo de duração de cada sessão (minutos) } \\
\hline 15 a 30 & 32 & 35,2 & 2 & 18,2 \\
\hline 40 a 60 & 50 & 54,9 & 4 & 36,4 \\
\hline Acima de 60 & 8 & 8,8 & 2 & 18,2 \\
\hline Não sabe & 1 & 1,1 & 3 & 27,3 \\
\hline \multicolumn{5}{|c|}{ Despendimento de quantia com exames diagnósticos } \\
\hline Sim & 44 & 48,4 & 2 & 18,2 \\
\hline Não & 46 & 50,5 & 8 & 72,7 \\
\hline Não sabe & 1 & 1,1 & 1 & 9,1 \\
\hline \multicolumn{5}{|c|}{ Interferência na atividade profissional a partir do tratamento da criança } \\
\hline Sim & 65 & 71,4 & 6 & 54,6 \\
\hline Não & 21 & 23,1 & 4 & 36,4 \\
\hline Não respondeu & 5 & 5,5 & 1 & 9,1 \\
\hline \multicolumn{5}{|c|}{ Oferecimento de algum tipo de assistência para a mãe } \\
\hline Sim & 50 & 54,9 & 4 & 36,4 \\
\hline Não & 41 & 45,1 & 7 & 63,6 \\
\hline
\end{tabular}

Fonte: Dados da pesquisa, 2019. 


\section{Discussão}

No que concerne ao perfil dos sujeitos, observa-se que a idade materna é superior a 21 anos de idade e a maior parte das mulheres não apresentam complicações durante o parto, de forma similar ao que foi encontrado no estudo de Marinho e colaboradores. ${ }^{\underline{7}}$

Com relação ao nível de escolaridade, a maioria das mães estudou menos que oito anos e, quanto à ocupação, dedicava-se ao lar e se auto referiram mães solteiras. A maior parte das famílias tinha renda familiar inferior a um salário mínimo e seu núcleo familiar era sustentado a partir do BPC. Identificou-se, assim, semelhança com os achados do estudo de Marinho et al. ․ㅡ na predominância da faixa etária materna mais jovem, na escolaridade e na condição econômica familiar, em que esses autores chamam atenção para as desigualdades socioeconômicas e geográficas das famílias das crianças que apresentam a Síndrome Congênita do Zika Vírus (SCZ).

A epidemia de Zika identificada no Nordeste brasileiro atingiu mulheres em idade reprodutiva. Foram mais afetadas as mães jovens, solteiras, com baixa escolaridade e em situação de pobreza. ${ }^{\text {? }}$

Não foi observada diferença de prevalência da microcefalia entre os sexos na amostra das crianças deste estudo. Além disso, o maior número dos cuidadores entrevistados eram as mães, evidenciando o que culturalmente já se sabe, a mulher é a provedora dos cuidados familiares, sobretudo ao se tratar do cuidado com crianças. .8 O papel da mãe no contexto familiar é de provedora do cuidado, quando se trata das que lidam com condições adversas, a exemplo das deficiências, o que as torna mais dependentes. Empoderadas pelo sentimento envolvido na maternidade e consciente de suas obrigações, as mães não restringem seus cuidados, podendo, por vezes, abdicar de outros aspectos de suas vidas. ? $^{-}$

Ao avaliar o momento em que a criança foi diagnosticada com microcefalia, salientou-se que mais da metade das mães teve diagnóstico após o parto.
Desse modo, o nascimento da criança foi acompanhado do impacto da notícia de que a criança apresentava uma deficiência, e lidar com isso projeta um sentimento de fracasso e frustração pela perda do filho idealizado e quebra das expectativas criadas pelos pais. Além disso, a deficiência de um filho traz consigo a necessidade de partilha das obrigações referentes ao cuidado entre os membros da família e o planejamento de novas possibilidades, inclusive terapêuticas, a fim de superar as adversidades. $\frac{3}{}$

O estudo em tela evidencia características do acesso ao tratamento fisioterapêutico, e em se tratando do domínio restrito do acesso, configurado pela busca pelo serviço e a entrada no mesmo. ${ }^{6}$ Identificou-se que quase a totalidade das crianças teve algum encaminhamento para a fisioterapia, e conseguiu acesso ao serviço de reabilitação. Todavia, outros estudos referem dificuldades em acessar o serviço, com uma entrada tardia, o que pode acarretar prejuízos para o estado de saúde do indivíduo.. Salienta-se que o acesso aos serviços de fisioterapia não parece ser uma situação facilmente vivenciada.

Analisando o contexto do acesso aos serviços de reabilitação no país, de modo geral, percebe-se que no caso das crianças com microcefalia houve uma diferenciação em termos da oferta e do acesso. O SUS ampliou o acesso aos cuidados de saúde da população brasileira, tendo o Ministério da Saúde (MS) proposto ações voltadas a esta problemática. Desde o ano de 2011 com o lançamento do Plano Viver Sem Limites, depois com a RCPCD, houve expressiva expansão dos serviços de reabilitação. Em 2015, com toda a repercussão gerada pelo problema da Síndrome Congênita do Zika Vírus (SCZV) e com forte clamor popular, o MS promoveu algumas ações visando atender às demandas dessas crianças e suas famílias e em 2015 lançou o Plano Nacional de Enfrentamento à Microcefalia. ${ }^{10}$ Em 2016, publicou o Protocolo de atenção à saúde relacionada à infecção pelo Zika Vírus, como também as Diretrizes de estimulação precoce - para crianças de zero a três anos com atraso no desenvolvimento neuropsicomotor decorrente de microcefalia. 
A promulgação da Convenção sobre os Direitos da Pessoa com Deficiência no Brasil é fruto de um processo de amadurecimento da sociedade, junto a este processo, em 2012 instituiu-se a Rede de Cuidados à Saúde da Pessoa com Deficiência no âmbito do SUS. A ampliação dos Centros Especializados em Reabilitação (CER) surge como um de seus avanços; conceituados como serviços de suporte que proporcionam a gestão de doenças neuromusculares e musculoesqueléticas que alteram o estado funcional, realizando intervenções clínicas, psicossociais e funcionais. 11 Verificou-se, no presente estudo que muitos dos casos estavam sendo acompanhados nos CER, os quais são preconizados como local especializado para o atendimento.

A maioria das crianças tiveram acesso ao tratamento fisioterapêutico imediato ou dentro do tempo ideal para o início da intervenção, fato observado também no estudo de Santos et al. ${ }^{12}$, em que a população estudada já fazia terapia em média há nove meses, o que demonstra o início precoce do tratamento e a adesão da família.

O acesso tardio, ou seja, em um período superior a 60 dias, foi identificado em um número menor de respondentes. Contudo a estimulação precoce é essencial para o desenvolvimento pós-natal do cérebro, pois a comunicação e conexão que ocorre entre as células corticais em um processo contínuo desencadeiam processos de aprendizagem, de acordo com cada fase, que podem perdurar para toda vida. ${ }^{13}$ Vale salientar que o início tardio da intervenção fisioterapêutica pode ocasionar o aparecimento de contraturas e deformidades, dores, diminuição da força muscular e demora na aprendizagem motora, dificultando a reintegração social do paciente..$^{14}$

Evidências da literatura especializada apontam que a reabilitação é um processo global e dinâmico. Ao iniciar o tratamento precocemente, potencializa-se a reorganização do tecido cerebral, maximizando assim a funcionalidade, ofertando recuperação da saúde física e psicológica da pessoa com deficiência. $\frac{14}{}$ A acolhida, atenção e o cuidado a essas crianças e a suas famílias são de grande relevância para que se alcance o maior ganho funcional possível nos primeiros anos de vida.
Assim, o fisioterapeuta no campo da reabilitação atua como potencializador de ganhos funcionais, auxilia na aquisição de marcos de desenvolvimento pelas crianças a partir da estimulação do funcionamento do SNC e de outras dimensões do funcionamento orgânico. $\frac{15}{\mathrm{O}}$ acesso à fisioterapia de qualidade é, portanto, um redutor de complicações físicas relacionadas às alterações articulares, de desempenho motor, tônus e força. As atividades motoras, simetria e amplitude, alteradas demandam a abordagem inicial do fisioterapeuta, com vistas a minimizar o impacto das sequelas motoras relacionados a SCZ, atuando na melhoria de sua independência funcional. ${ }^{16}$

Sabe-se que a microcefalia pode manifestar-se de modo peculiar em cada recém-nascido, podendo comprometer diversos sistemas do organismo, gerando desde problemas motores a respiratórios. Logo, o acompanhamento precoce e constante permite ainda um monitoramento da criança, de modo a fazer encaminhamentos especializados e promover uma assistência integral à saúde das crianças com microcefalia.

Tendo em vista o princípio da integralidade da assistência no SUS, segundo o qual o acesso a todos os níveis de atenção à saúde devem ser garantidos de modo a atender integralmente as necessidades de saúde, questiona-se o modo como estão sendo ofertados esses serviços, uma vez que alguns cuidadores referiram ter custeado o serviço de reabilitação em algum momento, por terem optado por realizar o tratamento em mais de um serviço, no público e em outro serviço privado.

No que diz respeito ao domínio amplo do acesso, no quesito continuidade do cuidado, observou-se que a maioria destas crianças, manteve-se em tratamento sem interrupções.

A criança com microcefalia deve ser estimulada e acompanhada diariamente para incitar o seu desenvolvimento neuropsicomotor, portanto a família é essencial para o desenvolvimento máximo da criança. ${ }^{13}$ Os sujeitos da pesquisa $(82,5 \%)$ têm acesso aos serviços de reabilitação há mais de 12 meses, sendo 41,7\% dos que se mantiveram em acesso com frequência de duas vezes na semana e $45,4 \%$ daqueles que em algum momento interromperam o tratamento. 
A constância com o tratamento de fisioterapia (88,3\%) se faz importante, uma vez que as crianças com microcefalia apresentam atraso no desenvolvimento neuropsicomotor, alterações visuais, auditivas, cognitivas e sensoriais, algumas podem ter transtornos no aparelho locomotor como pés tortos congênitos, luxação de quadril e artrogripose, impactando na independência funcional e na inserção social dessa população. ${ }^{17}$

O cuidado em reabilitação apresenta características peculiares que interferem no acesso. Ao citar a necessidade de tratamento após o nascimento da criança, a continuidade da assistência, a fim de alcançar melhora da capacidade funcional e da qualidade de vida. É importante destacar a especificidade de um cuidado fisioterapêutico contínuo, uma vez que as incapacidades destas crianças estão relacionadas com a presença de déficits motores, sensoriais e cognitivos e, que demandam tempo para otimização da funcionalidade. ${ }^{17}$ Esta assistência contínua justifica-se pelo fato de que a plasticidade neuronal perdura por vários anos, o que evidencia não existir um período limite para a finalização do tratamento de pacientes neurológicos em programas de reabilitação. $\frac{18}{}$

No entanto, o cuidado prolongado por sua vez, pode ter altos custos físicos, financeiros e emocionais o que pode levar a uma descontinuidade de acesso. Nesse sentido, refletir sobre "o abandono do serviço" ou a "descontinuidade no tratamento fisioterapêutico" $(11,7 \%)$ implica em analisar o acesso no domínio amplo proposto por Frenk ${ }^{6}$, o qual prevê a continuidade do cuidado.

A região Nordeste, considerada uma das mais pobres do Brasil, possui uma grande limitação no acesso amplo aos serviços de saúde. Os usuários citam como dificuldades para acessar os serviços: a grande espera para o atendimento às especialidades, a falta de transporte adequado, a distância dos serviços, a limitação de recurso financeiro e as limitações arquitetônicas nos serviços públicos e domicílios dos usuários. $\frac{19}{}$

Entende-se como acesso oportuno a capacidade de se obter um agendamento rápido, permitindo que o usuário obtenha assistência para condições agudas, já a continuidade é a capacidade do usuário acessar o profissional de saúde de referência para acompanhamento, tendo em vista uma relação duradoura com o profissional e, consequentemente, o cuidado. $\underline{20}$ Contudo, a necessidade de acesso destas crianças, se encontra para além do já discutido, cabe aqui identificar, portanto, a definição de acesso adequado.

Acesso adequado pode ser compreendido como o processo de cuidado contínuo, desde as condições de saúde, busca e entrada no serviço, até a criação de vínculo e adesão ao serviço, possibilitando a longitudinalidade do cuidado. Assim, torna-se adequado, ao atentar-se ao fluxo de encaminhamento, a marcação, ao horário de atendimento, ao suporte emocional e financeiro, familiar e governamental, a estrutura organizacional e operacional, aos recursos materiais e físicos, as redes de saúde, a qualificação profissional, a distribuição geográfica e a qualidade dos serviços, determinantes nas condições de permanência terapêutica.

Frente a interrupção ao tratamento, nos resultados apresentados na última tabela, não houve diferença na condição de oferta do acesso para que este fosse interrompido, nota-se, no entanto, algumas diferenças entre as respostas dadas pelos cuidadores das crianças que se mantiveram em acesso e àquelas que o interromperam em algum momento, dentre estes resultados, destaca-se o "tratamento ser realizado em outro município" e "apoio da rede de cuidados".

As dificuldades de transporte são consideradas obstáculos para o cuidado do filho, pois restringem o transporte para as atividades de reabilitação. De acordo com Félix e Farias ${ }^{21}$ a maioria das cidades com casos de Síndrome Congênita do Zika Vírus não tem atendimento especializado e por esse motivo pactuam com outros municípios, entretanto, às vezes, há a dificuldade em manter o transporte regular dessas crianças para o tratamento.

O estudo de Lima ${ }^{22}$ identificou que as mães vivenciaram estresse quando precisaram se deslocar para outro município na busca pela assistência à saúde de seus filhos. 
Outro dado importante versa sobre o apoio da rede de cuidados ao cuidador, Brunoni et al. .23 em seu estudo identifica que a nova rotina dos familiares da criança com microcefalia repercute de forma direta na qualidade de vida, além do acompanhamento da criança com microcefalia também é necessário direcionar cuidados especiais às mães e demais familiares. O suporte pode ser ofertado por profissionais da saúde, religião, família e assistência pública e privada ${ }^{24}$, porém, um grande número de mães, $63,6 \%$, que tiveram acesso descontinuado afirmaram não ter tido conhecimento sobre algum tipo de assistência.

Além das expectativas de melhora ou cura, as famílias são rodeadas por incertezas quanto ao futuro dos filhos e das ansiedades inerentes a condição da criança. 25 Somado a isso, a rotina diária do cuidado, as diversas pressões do dia a dia, na sua maioria, associadas à insegurança financeira ratificam a necessidade do apoio à criança e aos familiares.

Foi visto que as famílias passam por repercussões de ordem física, emocional e financeira, que surgem, provavelmente, devido ao abandono da atividade laboral por um dos genitores ou por ambos e pela dificuldade das famílias de manter-se com apoio satisfatório das instâncias governamentais ou de outras instâncias da sociedade e ainda pela readequação social da vida. Isso faz pensar que é importante que o poder público se debruce a ofertar programas de incentivos com foco tanto na assistência da criança quanto na manutenção da família que cuida do filho com microcefalia associado ao Zika Vírus.

O estudo limita-se por apresentar uma abordagem transversal, uma vez que o acompanhamento destas famílias, estudo de coorte, seria necessário para compreender se ao longo dos anos manteve-se o acesso ampliado.

\section{Conclusão}

A partir do presente estudo, foi possível identificar diversas potencialidades dos serviços com relação ao acesso das crianças com Microcefalia na Paraíba, o que difere do contexto geral dos estudos de acesso. Dentre as dificuldades, destacaram-se os casos de interrupção do tratamento, que podem estar relacionados às dificuldades de o tratamento fisioterapêutico acontecer no mesmo município, a problemas com transporte e/ou a falta de acolhimento com o processo de saúde/doença do cuidador. Em contrapartida, como potencialidades pôde-se perceber a partir da caracterização do acesso, que a maioria das crianças com microcefalia teve acesso contínuo à reabilitação motora, prioritariamente nos CER, o que denota a importância da ampliação da RCPCD, não obstante, é válido apontar a necessidade da qualificação profissional e do reconhecimento pelo serviço de qualidade prestado, independentemente da localidade.

Portanto, os resultados deste estudo podem corroborar para a ampliação do auxílio à essas crianças e seus cuidadores, a partir de políticas de públicas de expansão e planejamento de ações visando a qualidade destes serviços.

\section{Contribuições dos autores}

Farias DN participou da concepção, delineamento, análise estatística dos dados da pesquisa, interpretação dos resultados e redação do artigo científico. Ribeiro KSQS e Nascimento JA participaram da concepção, delineamento, busca e análise estatística dos dados da pesquisa, interpretação dos resultados e redação do artigo científico. Melo ES, Brito GEG e Neves RF participaram da concepção, delineamento e interpretação dos resultados. Melo ES, Lira LDB e Souza LMG participaram da coleta de dados da pesquisa e interpretação dos dados. Todos os autores participaram da revisão crítica do artigo e aprovação da versão final a ser publicada. 


\section{Conflitos de interesses}

Nenhum conflito financeiro, legal ou político envolvendo terceiros (governo, empresas e fundações privadas, etc.) foi declarado para nenhum aspecto do trabalho submetido (incluindo, mas não se limitando a subvenções e financiamentos, participação em conselho consultivo, desenho de estudo, preparação de manuscrito, análise estatística, etc.).

\section{Referências}

1. Menezes HLSM, Pacheco JN, Tomal NR, Guedes VR. Zica Vírus associado à microcefalia. Rev Pato Tocantis [Internet]. 2016;2(3):32-45. Disponível em: https://betas.uft.edu.br/ periodicos/index.php/patologia/article/download/1991/pdf

2. Von Der Hagen $M$, Pivarcsi $M$, Liebe J, Von Bernuth $H$, Didonato $\mathrm{N}$, Hennermann JB, et al. Diagnostic approach to microcephaly in childhood: a two-center study and review of the literature. Dev Med Child Neurol. 2014;56(8):732-41. https://doi.org/10.1111/ dmcn.12425

3. Dantas KO, Neves RF, Ribeiro KSQS, Brito GEG, Batista MC. Repercussões do nascimento e do cuidado de crianças com deficiência múltipla na família: uma metassíntese qualitativa. Cad. Saúde Pública. 2019;35(6):e00157918. https://doi. org/10.1590/0102-311X00157918

4. Tôrres AKV, Sarinho SW, Feliciano KVO, Kovacs MH. Acessibilidade organizacional de crianças com paralisia cerebral à reabilitação motora na cidade do Recife. Rev. Bras. Saúde Matern. Infant. 2011;11(4):427-36. https://doi.org/10.1590/S151938292011000400009

5. Travassos C, Viacava F. Acesso e uso de serviços de saúde em idosos residentes em áreas rurais, Brasil, 1998 e 2003. Cad Saúde Pública. 2007;23(10):2490-2502. https://doi.org/10.1590/S0102311X2007001000023

6. Travassos C, Castro MSM. Determinantes e desigualdades sociais no acesso e na utilização dos serviços de saúde. In: Giovanella L, Escorel S, Lobato LVC, Noronha JS, Carvalho Al. Políticas e sistema de saúde no Brasil. $2^{\mathrm{a}}$ ed. Rio de Janeiro: Editora FIOCRUZ; 2012. p. 183-206.

7. Marinho F, Araújo VEM, Porto DL, Ferreira HL, Coelho MRS, Lecca RCR, et al. Microcefalia no Brasil: prevalência e caracterização dos casos a partir do Sistema de Informações sobre Nascidos Vivos (Sinasc), 2000-2015. Epidemiol Serv Saude. 2016;25(4): 701-12. https://doi.org/10.5123/S167949742016000400004
8. Adegoke BOA, Adenuga OO, Olaleye OA, Akosile CO. Quality of life of mothers of children with cerebral palsy and their agematched controls. African J. Neurol. Sci [Internet]. 2014;33(1):355-61. Disponível em: https://www.ajol.info/index. php/ajns/article/view/115311

9. Gadelha IDS, Brito GE, Ribeiro KSQS, Nascimento JA, Freitas EML. Tempo de acesso aos serviços de reabilitação de pessoas acometidas por ave. Rev enferm UFPE [Internet]. 2015;9(12):124624. Disponível em: https://periodicos.ufpe.br/revistas/ revistaenfermagem/article/view/10831/12027

10. Ministério da Saúde (Brasil), Secretaria de Saúde. Febre pelo vírus Zika: uma revisão narrativa sobre a doença [Internet]. Brasília: Ministério da Saúde. 2015;46(26). Disponível em: http:// www.dive.sc.gov.br/conteudos/agravos/notas tecnicas/Vigilanciazika-31-01-17.pdf

11. Chen JJ, Yang RK. The Future of UIHC Rehabilitation Services: Defining and Measuring Quality Rehabilitation Services. Iowa Orthop J. 2009;29:139-42. Citado em: PMID: 19742103

12. Santos LS, Barbosa ASS, Santana ASFG, Monteiro LFT. A participação da família no trabalho de reabilitação da criança com microcefalia. Cad Grad Ciênc Biol Saúde Unit [Internet]. 2018;4(2):189-202. Disponível em: https://periodicos.set.edu.br/ fitsbiosaude/article/view/4525

13. Nunes ML, Carlini CR, Marinowic D, Kalil Neto F, Fiori HH, Scotta MC, et al. Microcephaly and Zika virus: a clinical and epidemiological analysis of the current outbreak in Brazil. J. Pediatr. 2016;92(3):230-40. https://doi.org/10.1016/j. jped.2016.02.009

14. Ribeiro AP, Barter EACP. Atendimento de reabilitação à pessoa idosa vítima de acidentes e violência em distintas regiões do Brasil. Ciênc. saúde coletiva. 2010;15(6):2729-40. https://doi. org/10.1590/S1413-81232010000600011

15. Ventura CV, Maia M, Ventura BV, Linden VVD, Araújo $\mathrm{EB}$, Ramos RC, et al. Achados oftalmológicos em lactentes com microcefalia e infecção presumida pelo vírus Zika. Arq. Bras. Oftalmol. 2016;79(1):1-3. https://doi.org/10.5935/00042749.20160002

16. Botelho ACG, Neri LV, Silva MQF, Lima TT, Santos KG, Cunha RMA, et al. Infecção congênita presumível por Zika vírus: achados do desenvolvimento neuropsicomotor - relato de casos. Rev. Bras. Saude Mater. Infant. 2016;16(1):39-44. https://doi. org/10.1590/1806-93042016005100004

17. Flor CJDRV, Guerreiro CF, Anjos JLM. Desenvolvimento neuropsicomotor em crianças com microcefalia associado ao Zika Vírus. Rev Pesq Fisioter. 2017;7(3):313-18. https://doi. org/10.17267/2238-2704rpf.v7i3.1386 
18. Silva RK, Gaetan ESM. A importância da estimulação ambiental e da intervenção fisioterapêutica precoce na habilitação de crianças com paralisia cerebral: uma visão neurofisiológica. Reabilitar. 2004;22(6):49-57.

19. Souza CCBX, Rocha EF. Portas de entrada ou portas fechadas? $\mathrm{O}$ acesso à reabilitação nas unidades básicas de saúde da região sudeste do município de São Paulo - período de 2000 a 2006. Rev Ter Ocup [Internet]. 2010;21(3):230-39. Disponível em: https:// pesquisa.bvsalud.org/portal/resource/pt/lil-657264

20. Balasubramanian H, Biehl S, Dai L, Muriel A. Dynamic allocation of same-day requests in multiphysician primary care practices in the presence of prescheduled appointments. Health Care Manag Sci. 2014;17(1):31-48. https://doi.org/10.1007/s10729$\underline{013-9242-2}$

21. Félix VPSR, Farias AM. Microcefalia e dinâmica familiar: a percepção do pai frente à deficiência do filho. Cad. Saúde Pública. 2018;34(12):e00220316. https://doi.org/10.1590/0102$311 \times 00220316$
22. Lima LHSS. Dinâmica familiar da criança com microcefalia pelo Zika vírus à luz da teoria de Betty Neuman [dissertação] [Internet]. Recife: Universidade Federal de Pernambuco; 2017. Disponível em: https://repositorio.ufpe.br/handle/123456789/29617

23. Brunoni D, Blascovi-Assis SM, Osório AAC, Seabra AG, Amato CADLH, Teixeira MCTV, et al. Microcefalia e outras manifestações relacionadas ao vírus Zika: impacto nas crianças, nas famílias e nas equipes de saúde. Ciênc. saúde coletiva. 2016;21(10):32973302. https://doi.org/10.1590/1413-812320152110.16832016

24. Bolla BA, Farias AMD. Cuidado da criança com anomalia congênita: a experiência da família. Esc Anna Nery Rev Enfermagem. 2013;17(2):284-90. https://doi.org/10.1590/S141481452013000200012

25. Vale PRLF. Experiências de famílias de crianças com microcefalia por Zika vírus [dissertação] [Internet]. Feira de Santana: Universidade Estadual de Feira de Santana; 2018. Disponível em: http://tede2.uefs.br:8080/handle/tede/680 